\title{
Mandenkan
}

MANDENIKAN Bulletin semestriel d'études linguistiques mandé

$50 \mid 2013$

Numéro 50

\section{L'espace déictique dans la langue mwan}

Deictic space in the Mwan language

ДЕЙКТИЧЕСКОЕ ПРОСТРАНСТВО В ЯЗЫКЕ МУАН

\section{Elena Perekhvalskaya}

\section{(2) OpenEdition}

Journals

Édition électronique

URL : https://journals.openedition.org/mandenkan/261

DOI : 10.4000/mandenkan.261

ISSN : 2104-371X

Éditeur

Llacan UMR 8135 CNRS/Inalco

\section{Édition imprimée}

Date de publication : 1 décembre 2013

Pagination : 103-116

ISSN : 0752-5443

\section{Référence électronique}

Elena Perekhvalskaya, «L'espace déictique dans la langue mwan », Mandenkan [En ligne], 50 | 2013, mis en ligne le 01 décembre 2013, consulté le 08 juillet 2021. URL : http://journals.openedition.org/ mandenkan/261 ; DOI : https://doi.org/10.4000/mandenkan.261

Ce document a été généré automatiquement le 8 juillet 2021.

\section{(c) (i) (2) (2)}

Les contenus de Mandenkan sont mis à disposition selon les termes de la Licence Creative Commons Attribution - Pas d'Utilisation Commerciale - Partage dans les Mêmes Conditions 4.0 International. 


\section{L'espace déictique dans la langue}

\section{mwan}

Deictic space in the Mwan language

ДЕЙКТИЧЕСКОЕ ПРОСТРАНСТВО В ЯЗЫКЕ МУАН

\section{Elena Perekhvalskaya}

\section{NOTE DE L'AUTEUR}

Cette recherche a été effectuée dans le cadre du Projet HШ-575.2012.6 (Programme présidentiel à l'appui des écoles scientifiques de premier plan « Ecole de linguistique générale Yu. S. Maslow ») et du Projet RGNF № 13-34-01015 « Les systèmes verbaux des langues mandé dans le contexte des recherches typologiques et aréales ».

\section{Abréviations}

$1 \mathrm{PL}=1^{\mathrm{e}}$ personne du pluriel

$1 \mathrm{SG}=1^{\mathrm{e}}$ personne du singulier

$2 \mathrm{PL}=2^{\mathrm{e}}$ personne du pluriel

$2 \mathrm{SG}=2^{\mathrm{e}}$ personne $\mathrm{du}$ singulier

$3 \mathrm{PL}=3^{\mathrm{e}}$ personne $\mathrm{du}$ pluriel

$3 S G=3^{e}$ personne $d u$ singulier

$\mathrm{ANAPH}=$ pronom anaphorique

ART $=$ article défini

CONJ = conjonctif

$\mathrm{COP}=$ copule

DAT = postposition à valeur dative

$\mathrm{E}=$ exemple elicié

$\mathrm{EMPH}=$ pronom de série emphatique

EXCL $=$ exclusif

$\mathrm{FOC}=$ marqueur de focus

FUT $=$ futur 


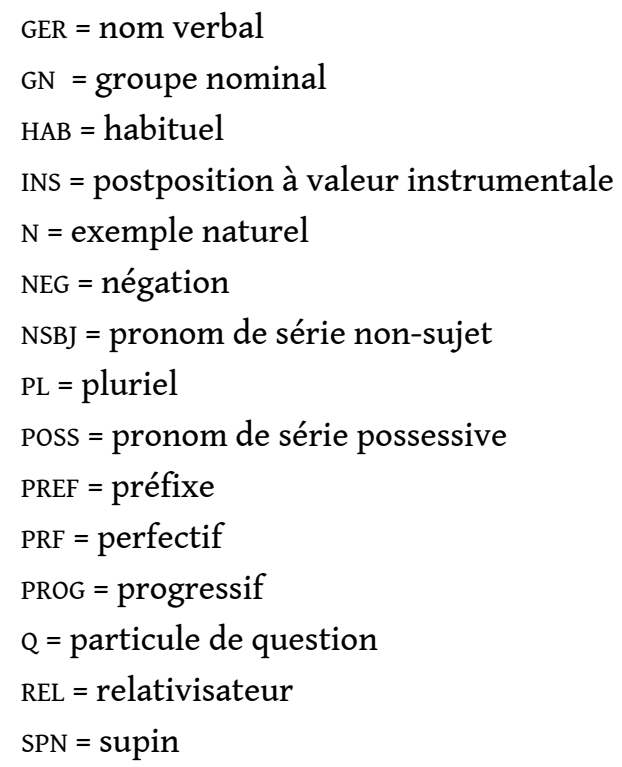

« Toute langue nous permet de conceptualiser l'espace qui nous entoure et de communiquer quelque chose sur lui » (Wassmann, Dasen 1998, 689).

1 Le centre des axes de coordonnées de l'espace linguistique est un acte de parole. «Dans toutes les langues naturelles, sans exception, les coordonnées linguistiques des faits sont données par rapport aux Lieu et Temps de l'acte de parole en d'autres termes par rapport à «ici » et «maintenant » (et, finalement, par rapport à «moi ») » (Mel'chuk 1998, II : 47).

2 Le Locuteur est placé dans l'espace du discours. Les principaux paramètres déictiques de l'acte de parole sont le «moi - ici - maintenant », nommé par Kasevich « la triade déictique de base » (Kasevich 1996 : 225). Suivant Espersen, Jakobson les a appelés « les mots-shifteurs ». La valeur de ces mots change avec chaque nouvel acte de parole, parce que chaque acte de parole a son propre, unique « moi », « ici » et « maintenant » (Jakobson 1971).

3 Dans cet article nous nous intéressons avant tout à « ici ». C'est le point de repère qui indique l'endroit où l'acte de la parole se produit et précise l'emplacement du Locuteur. Le Locuteur est placé au centre de l'espace, il représente le point à partir duquel l'espace déictique est mesuré.

\section{L'espace déictique dans les langues du monde}

Les coordonnées linguistiques sont inexactes ("diffuses») - les catégories de langue n'expriment jamais des valeurs exactes. La langue « est généralement basée sur des concepts très approximatifs... par exemple longue distance vs courte distance; devant vs derrière, jusqu'à (= avant) vs après, etc. » (Mel'chuk 1998, II : 48).

Dans les langues naturelles, les procédés déictiques se rapportent à l'espace du Locuteur. C'est la catégorie shifteur de deixis spatial. La position de l'objet se caractérise par sa proximité au Locuteur. Dans toutes les langues il y a des mots 
démonstratifs qui caractérisent la distance de l'objet par rapport au Locuteur (ex. français celui-ci - celui-là). C'est la division de l'espace « en termes de langue. »

Les langues divisent l'espace déictique différemment. La langue russe, de même que le français, l'anglais et de nombreuses autres langues, ont des systèmes à deux termes opposés selon l'axe "proche du Locuteur» vs «loin du Locuteur». Les systèmes binaires sont répandus mais pas du tout universels. Des nombreuses langues africaines, mwan inclus, présentent des systèmes bien plus compliqués.

7 En latin classique, il y a trois pronoms démonstratifs : hic 'ceci' indiquant des objets proches de la $1^{\text {re }}$ personne; iste 'ce', proche de la $2^{\mathrm{e}}$ personne, et ille 'cela' indiquant des objets proches de la $3^{\mathrm{e}}$ personne (Bourciez 1946 : 86). Le même modèle se manifeste en espagnol et en portugais. En espagnol, les trois membres du système déictique sont esto, eso et aquel. «Esto désigne un objet situé à proximité du Locuteur; eso est proche de l'Auditeur, aquello est loin de tous les deux » (Alonso, Ureña 1938, II : 120)1. Le système ternaire est assez répandu : on le trouve en tahitien, en japonais, etc.

8 Apparemment, le système des termes déictiques le plus compliqué parmi les langues naturelles comporte quatre éléments. La langue tabassaran peut en servir d'exemple.

Il y a d'autres systèmes déictiques compliqués, comme ceux de l'aléoutien (Golovko 1997), du nivkh (Gruzdeva 1997) et du tohono o'odham (Zepeda 1983), où d'autres paramètres entrent en jeu: la position de l'objet par rapport au Locuteur sur l'axe vertical ("plus haut» ou «plus bas»), la position de l'objet devant le Locuteur ou à côté ; la visibilité ou l'invisibilité de l'objet par le Locuteur, etc.

Une observation intéressante à propos du symbolisme acoustique est que les démonstratifs désignant l'espace proche du Locuteur contiennent normalement des voyelles fermées, tandis que les démonstratifs qui indiquent l'espace éloigné contiennent des voyelles ouvertes : E.g. angl. this - that; fr. ci - là, etc. (Plungian 2012 : $325-326)^{2}$.

11 Du point de vue catégoriel, les termes déictiques dans les langues du monde peuvent appartenir à des classes lexicales différentes : les adjectifs pronominaux (espagnol este, ese, aquel), les pronoms démonstratifs (russe eto - to), les adverbes spatiaux (anglais here - there), les verbes (bouriate iige- 'faire comme on le fait ici' - tiige- 'faire comme on le fait là'). Les adjectifs démonstratifs pronominaux et les pronoms démonstratifs constituent souvent une même classe de démonstratifs nominaux, selon le terme de R. M. W. Dixon (2003) : français ce livre est c'est (< ce est) un livre. Cette situation est typique des langues européennes, mais pas forcément des langues d'Afrique de Ouest, comme le mwan.

\section{L'espace déictique dans la langue mwan}

La langue mwan (groupe mandé sud) a un système déictique à six termes. Afin de définir le sémantisme de ces termes d'une façon exacte, j'ai entrepris une étude en utilisant une méthode élaborée par le groupe d'étude cognitive du langage de l'Institut d'Anthropologie Comparée de Max Planck (Levinson 1999).

13 Le but de ce travail est d'inventorier les procédés du deixis spatial en mwan et de les ranger selon les axes suivants :

1. les mots déictiques ayant le Locuteur pour point de repère et ceux ayant l'Adressé pour point de repère ; 
2. la distance (degrés d'éloignement par rapport au Locuteur) ;

3. autres paramètres du système déictiques : visibilité / invisibilité, contact physique / absence de contact physique ;

4. utilisation spécifique de chaque élément.

14 L'expérimentation s'est déroulée avec la participation de deux locuteurs natifs du mwan, tous les deux ayant une bonne pratique du français. Des objets différents (un livre, un téléphone portable, une chaise) ont été utilisés. On déplaçait ces objets et les mettait dans des positions différentes: en contact avec le corps du locuteur ou de l'auditeur; à des distances différentes par rapport aux participants de la communication; derrière ou devant un obstacle ; dans la position où l'objet était visible ou invisible du locuteur ou de l'auditeur, etc. L'informateur se présentait tantôt avec le rôle du locuteur, tantôt avec celui de l'auditeur. Il désignait l'objet en question en combinaison avec le mot en question (« cette chaise-ci », " cette chaise-là », etc.). On a demandé à l'informateur de créer un énoncé comportant l'expression déictique en question. Les résultats obtenus ont été vérifiés sur un corpus de textes mwan.

Dans cet article, les exemples naturels sont marqués par un $(\mathrm{N})$, et les exemples élicidés par un (E).

\section{Démonstratifs nominaux}

15 On trouve en mwan les démonstratifs nominaux suivants. Comme le système déictique français ne fournit pas d'équivalents qui pourraient représenter leur sémantisme d'une façon adéquate, j'utiliserai des index :

\begin{tabular}{|c|c|c|c|c|c|}
\hline léċ & $g \grave{\varepsilon}$ & $6 \bar{e}$ & $n \bar{u}$ & lāàte & $y \bar{e}$ \\
\hline $\mathrm{ce}_{1}$ & $\mathrm{ici}_{2}$ & $\mathrm{ici}_{3}$ & là ${ }_{1}$ & là ${ }_{2}$ & là ${ }_{3}$ \\
\hline
\end{tabular}

\section{Le positionnement des mots démonstratifs mwan dans le système des parties de discours}

16 Tous les termes déictiques mwan peuvent être utilisés comme des modificateurs nominaux, quelle que soit la partie du discours à laquelle ils appartiennent; ils constituent l'unique système déictique.

Un de ces lexèmes est un déterminant : léċ 'celui-ci'. Comme les autres déterminants

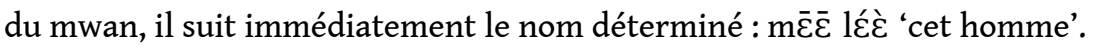

Les autres éléments du système déictique mwan sont des adverbes spatiaux. Cela se manifeste dans la façon dont ils sont connectés au groupe nominal qu'ils déterminent. Les adverbes spatiaux se connectent avec le GN par le moyen du relativisateur lá. Autrement dit, du point de vue formel, il s'agit de constructions relatives : mē $\bar{\varepsilon}$ lá gè 'l'homme qui (est) ci' mē $\bar{\varepsilon}$ lá bēe 'l'homme qui (est) là', etc.

19 À la différence du déterminant léċ, les adverbes spatiaux ne sont pas nécessairement contigus au nom déterminé. L'exemple (1) manifeste une construction relative de ce 
type où le nom déterminé et l'adverbe sont séparés par le circonstant é bà yí 'dans ta poche' :

\begin{tabular}{|c|c|c|c|c|c|c|c|c|c|c|c|}
\hline (1) & [Yée & pōtáblù & lá & $\{e ́$ & $b \grave{a}$ & $y i\}$ & $\boldsymbol{b} \bar{e}]$, & $\grave{e}$ & sว̄ & ý & ni. \\
\hline & 3SG.EMPH & portable & REL & $2 S G$ & poche & Dans & $\mathrm{ici}_{3}$ & $3 \mathrm{SG}$ & plaire & $1 \mathrm{SG}$ & DAT \\
\hline
\end{tabular}

Malgré des statuts syntactiques différents, les éléments déictiques forment l'unique système de modificateurs nominaux.

Considérons ces éléments un par un.

21 Le déterminant lé $\dot{\varepsilon}$ indique l'objet qui se trouve entre les mains du Locuteur, ou représente une partie de son corps, ou se trouve en contact immédiat avec le corps du Locuteur :

\begin{tabular}{|c|c|c|c|c|c|c|c|c|}
\hline (2) & $\hat{N}$ & $g b \bar{\varepsilon} n \grave{\varepsilon}$ & $6 \bar{\varepsilon}$ & léč, & $6 \varepsilon^{\prime}$ & làa & ǵ & $m \bar{a}$. \\
\hline & $1 S G$ & doigt & grain & $\mathrm{ce}_{1}$ & il & faire.souffrir & $1 \mathrm{SG}$ & sur \\
\hline & & là & & & & & & \\
\hline
\end{tabular}

\begin{tabular}{|c|c|c|c|c|}
\hline (3) & Blèe & léč́, & ỳé & trébó-zíi. \\
\hline & termite & $\mathrm{ce}_{1}$ & $3 S G>1 S G$ & irriter-PROG \\
\hline
\end{tabular}

Le déterminant léč apparaît également dans les cas où l'objet en question se trouve entre les mains de l'Adressé, dans le cas où le Locuteur indique cet objet avec son doigt et le touche. Il tombe donc dans la catégorie des objets qui se trouvent en contact immédiat avec le corps du Locuteur :

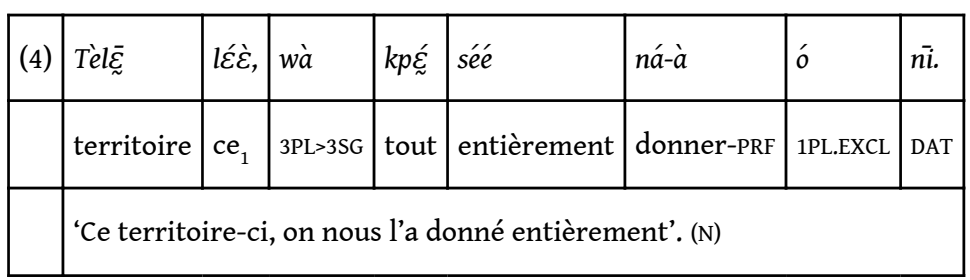

Le degré d'éloignement de l'objet de l'Adressé n'a aucune importance; l'objet peut être même hors de sa visibilité. Le déterminant léc̀ est employé même dans les cas où le Locuteur et l'Adressé sont séparés par une distance considérable, de façon qu'on est obligé de parler en élevant la voix où même en criant. 
24 Si le nom déterminé n'est pas une chose mais une notion abstraite, lćc̀ s'utilise pour indiquer que cette entité est proche dans le temps ou possède un statut pragmatiquement élevé :

\begin{tabular}{|l|l|l|l|l|l|}
\hline (5) & Ó & nuià & táá & léè & yí. \\
\hline & 3SG & arriver-PRF & fois & $\mathrm{ce}_{1}$ & dans \\
\hline \multicolumn{5}{|c|}{} \\
\multicolumn{4}{|c|}{ 'Nous sommes arrivés cette fois-ci'. (N) } \\
\hline
\end{tabular}

Apparemment, dans certains cas léc̀ perd sa relation avec le Locuteur, se transformant en marqueur de mise en relief :

\begin{tabular}{|c|c|c|c|c|c|c|c|c|c|}
\hline (6) & É & só & $g \bar{e}-\bar{l} \bar{e}$ & pegéé & niinà-lè & $k l \bar{\varepsilon}$-lé & $b i$ & léغ & $z \bar{i}$. \\
\hline & $2 S G$ & pouvoir-HAB & aller-GER & et & retourner-GER & faire-CONJ & nuit & $\mathrm{ce}_{1}$ & dans \\
\hline
\end{tabular}

La construction avec l'adverbe $g \grave{\varepsilon}$ indique l'objet qui se trouve à portée de main du Locuteur, mais hors du contact immédiat avec son corps :

\begin{tabular}{|l|l|l|l|l|l|l|l|}
\hline$(7)$ & Sćwé & lá & $g \grave{\varepsilon}$, & $y a ́$ & pía & $g b \bar{a}$ & ć̀े? \\
\hline & papier & REL & ici $_{2}$ & 2SG>3SG & lire.PRF & déjà & Q \\
\hline & $\begin{array}{l}\text { 'Est-ce que tu as déjà lu ce livre ?' (le livre dont on parle se trouve sur la table devant le } \\
\text { Locuteur). (E) }\end{array}$ \\
\hline
\end{tabular}

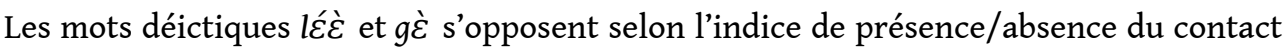
avec le corps du Locuteur. En même temps, les deux se réfèrent à une localisation dans la proximité immédiate du Locuteur.

La construction avec l'adverbe $\boldsymbol{b} \overline{\boldsymbol{e}}$ indique l'objet qui se trouve dans l'espace proche de l'Adressé, avec ou sans contact immédiat avec son corps. Le degré d'éloignement par rapport au Locuteur n'est pas pertinent: l'objet indiqué par la construction avec l'adverbe $b \bar{e}$ peut se trouver hors d'atteinte, peu visible où même invisible pour le Locuteur.

\begin{tabular}{|c|c|c|c|c|c|c|c|c|}
\hline (8) & É & 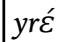 & kpá & blèe & lá & bè & $\grave{a}$ & $t \bar{a}$. \\
\hline & $2 S G$ & œil & mettre & termite & REL & $\mathrm{ici}_{3}$ & $3 S G$ & sur \\
\hline
\end{tabular}




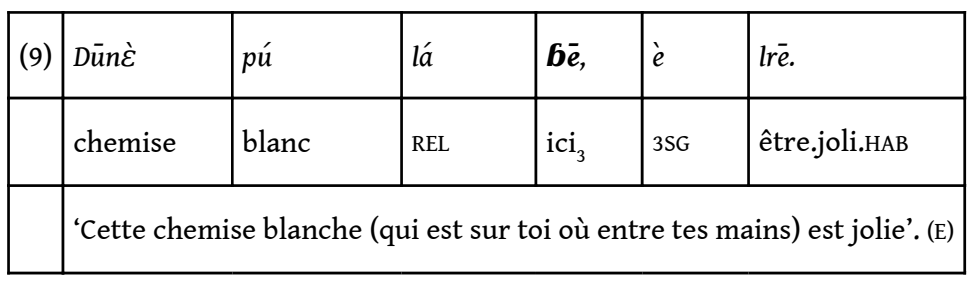

L'adverbe $b \bar{e}$ apparaît également quand l'objet indiqué est situé à une distance égale entre le Locuteur et l'Adressé :

\begin{tabular}{|l|l|l|l|l|l|l|l|}
\hline$(10)$ & Sćwé & lá & $\mathbf{G} \overline{\boldsymbol{e}}$, & $\grave{e}$ & $s \bar{\jmath}$ & $\dot{\eta}$ & $\overline{n i .}$ \\
\hline & papier & REL & $\mathrm{ici}_{3}$ & $3 \mathrm{SG}$ & plaire & $1 \mathrm{SG}$ & DAT \\
\hline & $\begin{array}{l}\text { 'Ce livre me plaît' (le livre se trouve entre le Locuteur et l'Adressé à la portée des mains de } \\
\text { chacun). (E) }\end{array}$ \\
\hline
\end{tabular}

Bè peut s'utiliser même si le Locuteur ne voit pas l'Objet, à condition que le Locuteur connaisse l'objet en question.

31 Ainsi, le déterminant léc̀ et l'adverbe gè ont la $1^{\text {re }}$ personne (le Locuteur) pour point de repère ; l'adverbe $\bar{b} \bar{e}$ a la $2^{\mathrm{e}}$ personne (l'Adressé) comme point de repère, le facteur de présence ou d'absence du contact avec le corps de celui-ci n'étant pas pertinent. Nous voyons que « l'espace du Locuteur » s'avère plus détaillé que « l'espace de l'Adressé ».

L'adverbe $\bar{b} \bar{e}$ apparaît également dans la situation où l'objet se trouve à une courte distance, mais s'avère "plus aliéné» par rapport aux deux autres procédés susmentionnés. Ainsi, il peut indiquer l'objet en possession d'une tierce personne, si cette personne est proche des interlocuteurs. L'énoncé tásá lá bee 'cette cuvette' peut indiquer qu'il s'agit d'une cuvette entre les mains d'une tierce personne si cette personne se trouve dans l'espace commun avec les interlocuteurs. E.g. :

\begin{tabular}{|l|l|l|l|l|l|l|l|l|l|}
\hline (11) & Yée & [bútèli & lá & $\{a$ & $k$ ḱó\} & Gēe $]$ & a & pá-le & ò. \\
\hline & 3SG.EMPH & bouteille & REL & 3SG.NSBJ & main & ici $_{3}$ & 3SG.NSBJ & remplir-GER & COP \\
\hline \multicolumn{10}{|c|}{} \\
\hline
\end{tabular}

Ici la phrase relative búteli lá bè 'bouteille qui est là' contient le circonstant à kóó 'entre ses mains'.

C'est aussi la construction avec l'adverbe bē qui apparaît dans le cas où l'objet ne se trouve pas loin "mesuré en valeur absolue ", s'il appartient à un autre "quantum d'espace ».

\begin{tabular}{|l|l|l|l|l|l|}
\hline$(12)$ & Kpè & lá & bè & $\grave{e}$ & sì̀-là. \\
\hline & chaise & REL & ici $_{3}$ & $35 \mathrm{SG}$ & casser-PRF \\
\hline
\end{tabular}


'Cette chaise est cassée’. (E)

Il s'agit de la chaise qui est à côté des interlocuteurs, plus ou moins à portée de leurs mains, mais séparée d'eux par une porte du balcon, c'est-à-dire dans «un espace aliéné ».

La construction avec l'adverbe déictique $n \bar{u}$ s'utilise par rapport à un Objet qui se trouve près du Locuteur (à une distance à peu près égale à celle indiquée par l'adverbe $g \grave{\varepsilon}$ ), mais très éloigné de l'Adressé où même invisible pour lui (par exemple, dans la situation d'une conversation téléphonique) :

\begin{tabular}{|c|c|c|c|c|c|c|}
\hline (13) & Tásá & lá & $n \bar{u}$ & yò-ò & $e ́-p u ́$ & lé. \\
\hline & cuvette & REL & là & 3SG-COP & PREF-blanc & INS \\
\hline
\end{tabular}

Cette phrase a été obtenue par l'élicitation. Je n'ai trouvé aucun exemple de l'utilisation de cet élément déictique en fonction de modificateur nominal dans les textes naturels. L'élément $n \bar{u}$ est d'emploi rare, et dans mon corpus il n'apparait qu'en tant qu'adverbe spatial.

38 Tous les mots déictiques examinés jusqu'ici se réfèrent aux objets qui se localisent dans l'espace proche du Locuteur. Pour les objets qui se trouvent dans l'espace éloigné, on utilise la construction avec l'adverbe lāà lé.

Cet adverbe indique un Objet qui se trouve hors d'atteinte des interlocuteurs, le plus souvent à une distance considérable :

\begin{tabular}{|c|c|c|c|c|c|c|c|}
\hline \multirow[t]{2}{*}{ (14) } & $F \varepsilon$ & lá & làāté, & $\grave{e}$ & s̄̄ & $\dot{y}$ & ni. \\
\hline & maison & REL & là ${ }_{2}$ & $3 S G$ & plaire & $1 \mathrm{SG}$ & DAT \\
\hline & $\begin{array}{l}\text { 'Cette } \mathrm{m} \\
\text { bien la v }\end{array}$ & qui e & e plaî & naiso & גve à u & nètre & ais on peut \\
\hline
\end{tabular}

La construction avec l'adverbe lāàte peut indiquer un Objet qui n'est pas trop éloigné, mais se trouve dans l'espace de la troisième personne, si cette personne ne partage pas l'espace commun du Locuteur et de l'Adressé :

\begin{tabular}{|c|c|c|c|c|c|c|c|c|c|c|}
\hline (15) & $\grave{E}$ & Lyéē & pōtáblù & lrè & lá & lāàtè] & $6 \varepsilon ́$ & $y \hat{a}$ & náa & téċe? \\
\hline & $3 S G$ & 3SG.ANAPH & portable & beau & REL & là & 3SG.ANAPH & voir.PRF & où & Q.intéressant \\
\hline
\end{tabular}

41 L'adverbe lāàtē correspond donc au degré suivant dans la hiérarchie d'éloignement. 
La construction avec l'adverbe $\bar{y} \overline{\boldsymbol{e}}$ indique un Objet qui se trouve hors de la vue du Locuteur et de l'Adressé. Ainsi, le sémantisme de cet élément inclut un paramètre supplémentaire, « la visibilité/invisibilité » de l'objet $^{3}$, e.g. :

\begin{tabular}{|c|c|c|c|c|c|c|c|}
\hline (16) & Làklūù & wà & lá & $y \bar{e}$ & yò-ò & 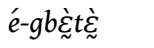 & lé. \\
\hline & école & village & REL & là & 3SG-COP & PREF-grand & INS \\
\hline & \multicolumn{7}{|c|}{ 'Cette université là-bas (nous ne la voyons pas) est grande’. (E) } \\
\hline
\end{tabular}

Si les interlocuteurs se déplacent dans un endroit d'où on peut voir l'objet en question, l'adverbe yè est remplacé par lāàte : làklūù wà lá lāâlêe... 'Cette université là-bas (nous la voyons de loin)...'

Les mots déictiques examinés peuvent être représentés dans le tableau 1.

Tableau 1. Les termes démonstratifs mwan

\begin{tabular}{|c|c|c|c|c|c|c|}
\hline & léc̀ & $g \grave{\varepsilon}$ & $6 \bar{e}$ & $n \bar{u}$ & lāàtè & $y \bar{e}$ \\
\hline proche du Locuteur & + & + & - & + & - & - \\
\hline proche de l'Adressé & - & - & + & - & - & - \\
\hline contact avec le corps & + & - & & - & - & - \\
\hline éloigné & - & - & - & - & + & + \\
\hline invisible & - & - & - & + à l'Adressé & - & + \\
\hline
\end{tabular}

44 Comme le montre le tableau 1, le mwan distingue trois degrés d'éloignement qui correspondent aux trois personnes grammaticales. A quoi s'ajoutent des paramètres additionnels : le contact avec le corps et la visibilité/invisibilité.

\section{Adverbes déictiques spatiaux}

À l'exception du déterminant lés̀, tous les éléments déictiques peuvent s'utiliser, et en fait s'utilisent le plus souvent en fonction adverbiale (fonction du circonstant) en

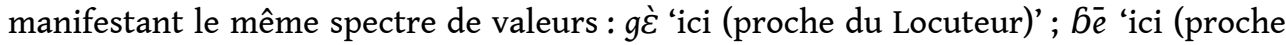
de l'Adressé)' ; nū 'ici, là (proche du Locuteur, loin de l'Adressé)'; lāàte 'là-bas (loin des interlocuteurs)' ; yè 'là-bas (très loin, invisible)'.

Pour les éléments les plus fréquents, $g \grave{\varepsilon}$ (espace proche du Locuteur) et $b \bar{e}$ (espace proche de l'Adressé), il est parfois difficile de distinguer entre leurs emplois dans le sens spatial et temporel (ou autres sens non-déictiques). Ce sont ces deux éléments qui apparaissent le plus souvent dans les énoncés copulatifs et dans les expressions lexicalisées sááani gè et sááníi bē 'maintenant'. 
Cependant, une distinction se manifeste : $g \grave{\varepsilon}$ indique souvent l'endroit proche ou lié à la $1^{\text {re }}$ personne, tandis que $b \bar{e}$ est lié à la $2^{\mathrm{e}}$ personne', ex. :

\begin{tabular}{|l|l|l|l|l|l|l|l|l|}
\hline$(17)$ & Ké & ká & láá & níinā-l $\bar{\varepsilon}$ & $g \grave{\varepsilon}$, & $o$ & $k a ́$ & $d \bar{\varepsilon}$. \\
\hline & si & $2 \mathrm{PL}$ & $\mathrm{NEG}$ & retourner-SPN & $\mathrm{ici}_{2}$ & $1 \mathrm{PL} . \mathrm{EXCL}$ & $2 \mathrm{PL}$ & tuer \\
\hline \multicolumn{8}{|c|}{ 'Si vous ne retournez pas ici (où nous sommes), nous vous tuerons'. (N) } \\
\hline
\end{tabular}

L'adverbe $b_{\bar{e}}$ indique l'endroit où se trouvent des Auditeurs (groupe de personnes).

\begin{tabular}{|l|l|l|l|l|l|l|l|l|l|l|}
\hline (18) & Ké & é & $d \bar{\varepsilon}$ & $\grave{o}$ & $6 \bar{e}$, & $k e$ & $y \grave{a}$ & pè-à & é & $\overline{n i}$ \\
\hline & et & $2 \mathrm{SG}$ & Père & COP & ici $_{2}$ & et & $3 \mathrm{SG}>3 \mathrm{SG}$ & dire-PRF & $2 \mathrm{SG}$ & DAT \\
\hline
\end{tabular}

\begin{tabular}{|l|l|l|l|l|l|}
\hline$z \grave{a}$ & $l a ́$ & $m \bar{a}$ & $y a ́ a ́$ & $k l \bar{\varepsilon}-l \bar{\varepsilon}$ & $b \bar{e} . .$. \\
\hline affaire & REL & à & 2SG.NEG & faire-SPN & ici $_{2}$ \\
\hline & \multicolumn{3}{|r}{ 'Si tu as un père, et s'il te dit de ne pas faire quelque chose là...' (N) } \\
\hline
\end{tabular}

Les valeurs des autres éléments déictiques peuvent être définies plus précisément.

$N \bar{u}$ indique un endroit familier au Locuteur, mais inconnu de l'Adressé. La position exacte de cet endroit n'est pas clarifiée. Il se comporte comme un marqueur de la spécificité pragmatique (Givón 1984), cf. russe odin 'un (certain)' : odin moj drug 'un ami à moi'.

\begin{tabular}{|l|l|l|l|l|l|}
\hline$(19)$ & $M i$ & $g b u ́$ & $\grave{o}$ & $n \bar{u}$ & $z \grave{\nu}$. \\
\hline & 1SG.EMPH & même & CoP & là & aujourd'hui \\
\hline & \multicolumn{3}{|c|}{ 'Moi-même, j'ai été à un certain endroit aujourd'hui.' (N) } \\
\hline
\end{tabular}

51 Le Locuteur a en vue un endroit précis, mais il ne trouve pas nécessaire de le nommer.

52 Lāâte s'utilise pour indiquer la distance éloignée, mais accessible: c'est plutôt «à l'extrémité du village » qu' «à Abidjan »(dans ce dernier cas, on emploierait yē ).

\begin{tabular}{|l|l|l|l|}
\hline$(20)$ & Yée & bàláá & lāàlè. \\
\hline & 3SG.EMPH & tomber-PRF & là \\
\hline & 'Lui, il tomba là-bas (loin d'ici). (N) \\
\hline
\end{tabular}


$Y_{\bar{e}}$ désigne un endroit qui se trouve très loin du centre déictique, par exemple, au Canada, en France : il ne suffit pas que la distance soit considérable, il faut également qu'il s'agisse d'un endroit étranger, ex. :

\begin{tabular}{|c|c|c|c|c|c|c|c|c|c|c|}
\hline (21) & Béć & ò & bō & $y \bar{e}$ & ò & $g \bar{a} \bar{a} \bar{a}$ & $z \bar{a}$ & $y \hat{i}$ & srỡ & léć. \\
\hline & puis & 3PL & rester-НАВ & $\operatorname{là~}_{3}$ & 3Pl & cacher & cause & dans & sorcellerie & devant \\
\hline
\end{tabular}

\section{La combinaison de démonstratifs différents}

La combinaison de démonstratifs différents permet d'exprimer des valeurs pragmatiques :

\begin{tabular}{|c|c|c|c|c|c|c|c|}
\hline (22) & BÉċyíle-é & ó & $g \grave{u}-\grave{a}$ & $y \bar{e}$. & ń & ná & $6 \varepsilon$ \\
\hline & c'est pourquoi & 1PL.EXCL & quitter-PRF & là & $1 S G$ & femme & 3SG.ANAPH \\
\hline
\end{tabular}

\begin{tabular}{|c|c|c|c|c|c|c|c|}
\hline bù̀à & Zènúflā & $\boldsymbol{b} \bar{e}$ & $s \bar{a}$ & 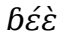 & $m i ́$ & $n \grave{u}-\grave{a}$ & gì. \\
\hline rester.PRF & Zouénoula & $\mathrm{ici}_{3}$ & un.peu, & et & 1SG.EMPH & venir-PRF & $\mathrm{ici}_{2}$ \\
\hline & \multicolumn{7}{|c|}{$\begin{array}{l}\text { 'C'est pourquoi nous sommes partis de là (du village qui se trouve très loin). Ma femme, } \\
\text { elle restait un peu là (chez elle) à Zouénoula, et moi, je suis venu ici (où je suis } \\
\text { maintenant)'. (N) }\end{array}$} \\
\hline
\end{tabular}

Dans cet énoncé, l'adverbe $b e ̄ e q u i$ indique l'espace "du deuxième ordre ", indique la ville natale de la femme du Locuteur. Le village que le Locuteur avait quitté était dans la région occupée par des forces rebelles; c'était un « espace étrange ».

Parmi les adverbes déictiques, certains peuvent se combiner :

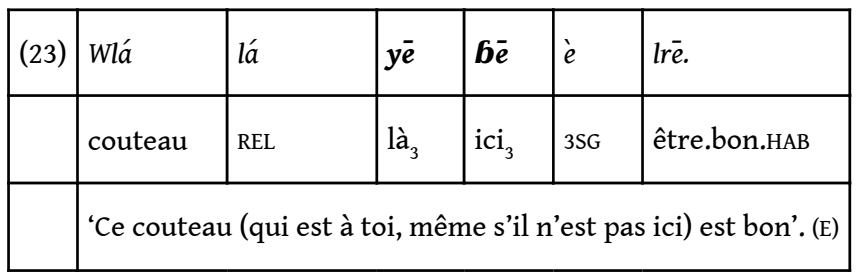

Dans les combinaisons des démonstratifs, d'habitude, l'un exprime une valeur démonstrative, et l'autre représente d'autres types de valeurs dont il sera question cidessous. 

$g \grave{\varepsilon} b_{\bar{e}}^{\bar{e}}$ et ye $\bar{b} \bar{e}$ ont été trouvées. Assez souvent, ils font partie de la construction présentative :

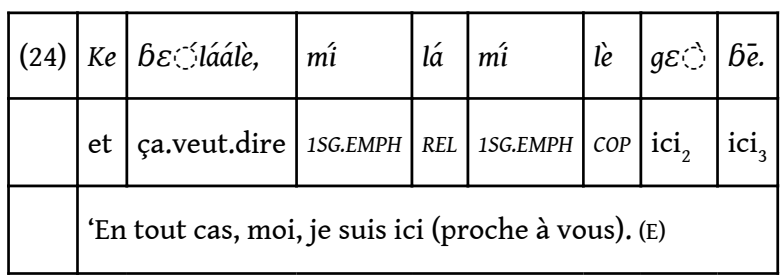

En (24), le premier élément ge fait partie de la construction présentative, tandis que bè est l'adverbe spatial. Dans l'exemple suivant (25) gE fait partie de l'expression lexicalisée sáání gè 'maintenant' :

\begin{tabular}{|c|c|c|c|c|c|c|c|c|}
\hline (25) & BÉ $\varepsilon^{\prime}-:$ & $\grave{o}$ & $b \bar{o}$ & $y \bar{e}$ & srỡ & $z \grave{a}$ & $\varepsilon$ & k \\
\hline & puis & 3PL & rester-HAB & là & sorcellerie & affaire & ART & chez \\
\hline
\end{tabular}

\begin{tabular}{|l|l|l|l|}
\hline dúy & sááíi & $g \mathcal{E}_{\text {à }}$ & $6 \bar{e}$. \\
\hline mais & maintenant & $\mathrm{ici}_{2}$ & $\mathrm{ici}_{3}$ \\
\hline & 'Et puis ils restent là-bas à cause de la sorcellerie donc maintenant là'. (N) \\
\hline
\end{tabular}

Cependant, la fréquence de ces éléments dans les textes varie considérablement. Dans le corpus des textes mwan de 16300 mots, la fréquence des tous les éléments déictiques est la suivante ${ }^{4}$ :

Tableau 2. Fréquence des éléments démonstratifs dans le corpus des textes mwan

\begin{tabular}{|l|l|l|l|l|l|l|l|}
\hline léč & gè & bē & nū & lāālē & yē & gè bē & yē bē \\
\hline 43 & 126 & 256 & 12 & 9 & 40 & 17 & 3 \\
\hline $8,5 \%$ & $24,8 \%$ & $50,6 \%$ & $2,4 \%$ & $1,8 \%$ & $7,9 \%$ & $3,4 \%$ & $0,6 \%$ \\
\hline
\end{tabular}

\section{Autres fonctions des termes déictiques}

61 Les adverbes déictiques $g \grave{\varepsilon}, b \bar{e}, y \bar{e}, n \bar{u}$ peuvent apparaitre à titre d'éléments adverbiaux dans les constructions copulatives et autres qui requièrent une composante adverbiale. Dans ce contexte, l'adverbe g̀̀ s'avère le plus neutre et presque désémantisé. Cet adverbe apparaît également dans les énoncés hors contexte, sans liens déictiques avec le discours. Il s'agit tout d'abord des constructions avec la copule présentative lè : 


\begin{tabular}{|l|l|l|l|}
\hline$(26)$ & $Z \bar{\varepsilon}$ & $\grave{l}$ e & $g \grave{\varepsilon}$. \\
\hline & rônier & CoP & ici $_{2}$ \\
\hline & \multicolumn{3}{|c|}{ 'C'est un rônier'. (N) } \\
\hline
\end{tabular}

\begin{tabular}{|l|l|l|l|l|l|l|l|}
\hline$(27)$ & $A$ & $\bar{t} \bar{i}$ & $g \bar{a}-\bar{l} \bar{l}$ & $l \bar{\varepsilon} \bar{\varepsilon}$ & $p l \bar{\varepsilon}$ & $l \grave{e}$ & $g \grave{\varepsilon}$. \\
\hline & 3SG.NSBJ & oncle.maternel & mourir-GER & année & deux & COP & ici $_{2}$ \\
\hline \multicolumn{7}{|l}{} \\
\hline \multicolumn{7}{|l}{ 'Son oncle maternel est mort depuis deux ans'. (E) } \\
\hline
\end{tabular}

L'élément adverbial est requis également pour exprimer la corrélation temporelle des énoncés :

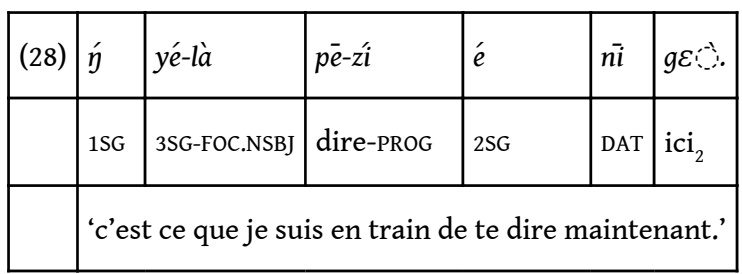

En effet, les autres adverbes déictiques s'emploient aussi dans les énoncés nonverbaux :

\begin{tabular}{|l|l|l|l|}
\hline$(29)$ & Wàă $\bar{a} \bar{a} \bar{a}$ & $\grave{o}$ & $y \bar{e}$. \\
\hline & Dieu & Cop & là \\
\hline & \multicolumn{2}{|c|}{ 'Dieu existe' (mais il est invisible). (N) } \\
\hline
\end{tabular}

L'adverbe $g \grave{\varepsilon}$ apparaît en tant que substitut d'un mot oublié :

\begin{tabular}{|c|c|c|c|c|c|c|c|c|c|c|}
\hline (30) & o & $g \grave{\varepsilon} .$. & láá & $z \bar{i} \bar{i} \bar{\Sigma}$ & wésé & láá & $\overline{z i} \bar{\sim} \bar{\sim}$ & $n \grave{a}-\grave{a}$ & $\dot{y}$ & $\overline{n i}$. \\
\hline & 3PL & ici $_{2}$ & feuille & sauce & patate & feuille & sauce & donner-PRF & $1 \mathrm{SG}$ & DAT \\
\hline
\end{tabular}

Dans ce cas l'adverbe peut avoir des marqueurs correspondants, comme la marque du pluriel ou l'article :

\begin{tabular}{|c|c|c|c|c|c|c|c|c|c|}
\hline (31) & Ké & wóo... & $g \varepsilon_{0}$ & $m \bar{u}$ & 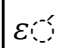 & pwe & sàyāà & $m \bar{u}$ & $\varepsilon$ \\
\hline & et & 3PL.EMPH & $\mathrm{ici}_{2}$ & PL & ART & entrer-CONJ & bandit & PL & ART \\
\hline
\end{tabular}


'Et ils... le machin... les bandits sont venus'. (N)

\section{Conclusion}

À la différence de la majorité des langues du monde qui distinguent nettement entre les démonstratifs de type adjectival et les adverbes spatiaux, le mwan a un système mixte. A part lés qui fonctionne comme un déterminant, tous les autres éléments sont des adverbes spatiaux dont le fonctionnement se rapproche de celui des démonstratifs nominaux lorsqu'ils apparaissent dans le cadre d'une proposition relative. Cette fonction est évidemment secondaire : les noms déterminés par de telles propositions relatives sont en même temps mis en relief.

Le système déictique du mwan distingue trois degrés d'éloignement, ce qui est compliqué par deux paramètres supplémentaires: visibilité / invisibilité, contact physique / absence de contact physique.

\section{BIBLIOGRAPHIE}

ALONSO Amado, UREÑA Pedro.H., 1938, Gramática castellana, T. I-II, Buenos Aires, Losada.

BOURCIEz Edouard, 1946, Éléments de linguistique romane, (4e édit.), Paris,G. Klincksieck.

DIXON, R.M.W., 2003, “Demonstratives: A cross linguistic typologie”, Studies in languages 27:1, pp. 61-113.

GIVÓN Talmy, 1984, “The pragmatics of referentiality”, in Deborah SCHRIFFIN (ed.) Geogetown University Round Table on language and linguistics, Washington D.C., Georgetown University Press, pp. 120-38.

GolovKo Evgenij V., 1997, « La langue aléoutienne » [Aleutskij jazyk], in Jazyki mira. Paleoaziatskije jazyki, Moskva, Indrik, pp. 101-116.

GRUZDEVA Ekaterina Ju., 1997, « La langue nivkh » [Nivkhskij jazyk], in Jazyki mira. Paleoaziatskije jazyki, Moskva, Indrik, pp. 139-155.

JAKOBSON Roman, 1971, "Shifters, verbal categories, and the Russian verb", in Selected writings II, The Hague, Mouton, pp. 130-147.

KASEVICH Vadim B., 1996, Le bouddhisme. L'image du monde. Langue. [Buddizm. Kartina mira. Jazyk], Sankt-Peterburg, Peterburgskoje vostokovedenije.

LEVINSON Stephen C., "Deixis and demonstratives”, in David WILKINS (ed.), Manual for the 1999 Field Season, pp. 29-40. Nijmegen: Max Planck Institute for Psycholinguistics.

MEILLET Antoine, 1938, Introduction à l'étude comparative des langues indo-européennes, Paris, Hachette. 
MEL'CHUK Igor A., 1998, Cours de morphologie générale, Vol. II (traduction russe) [Moskva: Jazyki russkoj kultury] Wien, Wiener Slavistischer Almanach.

PLUNGIAN Vladimir A., 2012, Le sémantisme grammatical : les valeurs grammaticales et les systèmes grammaticaux des langues du monde [Grammaticheskaya semantika: grammaticheskie znacheniya $i$ grammaticheskie sistemy yazykov mira], Moskva, RGGU.

WASSMANN Jurg, DASEN Pierre R., 1998, “Balinese spatial orientation: some empirical evidence of moderate linguistic relativity", Journal of the Royal Anthropological Institute 4, pp. 689-711.

ZEPEDA Ofelia, 1983, A Tohono O'odham grammar, Tucson, University of Arizona Press.

\section{NOTES}

1. Antoine Meillet mentionne l'existence des plusieurs racines avec une valeur démonstrative dans la protolangue indo-européenne (Meillet 1938: 333). On peut faire l'hypothèse que cette langue aussi avait un système d'espace déictique à trois termes.

2. Il est intéressant de voir que les langues mandingues contredisent ce principe, cf. bambara yàn 'ici' - yèn 'là'.

3. En fait, l'élément précédent (lāà ēe est impliqué dans l'opposition «visible/invisible» également; il y est marqué positivement.

4. Toutes les occurrences ont été calculées : celles des déterminants et des adverbes spatiaux. Les combinaisons des deux éléments ont été comptées séparément.

\section{RÉSUMÉS}

L'article traite d'un système compliqué des démonstratifs en mwan (Mandé-sud). L'étude est basée sur les données expérimentales obtenues en utilisant une méthode élaborée par un groupe de recherche de linguistique cognitive de Max Planck. Les propriétés morphologiques, syntaxiques et fonctionnelles des démonstratifs en mwan ont été analysées. Il s'avère que le système des démonstratifs en mwan comporte cinq éléments qui s'opposent par des indices suivants: 1) le locuteur, l'auditeur ou une tierce personne comme le point de repère; 2) localisation dans la vue ou hors la vue du locuteur ou de l'auditeur ; 3) localisation en contacte ou hors contacte avec le corps du locuteur.

The article deals with a rather complicated system of demonstratives in Mwan (South Mande). The research was based on the experimental data obtained while using the methodology elaborated by de Max Planck Research group of Cognitive linguistics. Morphological, syntactic and functional proprieties of the Mwan demonstratives are analysed. It is shown that the Mwan system of demonstratives consists of five elements which can be: 1) Speaker, Adressee or Third Person anchored; 2) within or out of sight of Speaker or Adressee; 3) in or out of contact with Speaker's body.

В СТАТЬЕ РАССМАТРИВАЕТСЯ ДОВОЛЬНО СЛОЖНАЯ СИСТЕМА ДЕМОНСТРАТИВОВ ЯЗЫКА МУАН (ЮЖНАЯ ГРУППА МАНДЕ). ИССЛЕДОВАНИЕ ОСНОВЫВАЕТСЯ НА ЭКСПЕРИМЕНТАЛЬНЫХ ДАННЫХ, 
ПОЛУЧЕННЫХ С ПРИМЕНЕНИЕМ МЕТОДИКИ, РАЗРАБОТАННОЙ ИССЛЕДОВАТЕЛЬСКОЙ ГРУППОЙ КОГНИТИВНОЙ ЛИНГВИСТИКИ ИНСТИТУТА МАКСА ПЛАНКА. РАССМАТРИВАЮТСЯ МОРФОЛОГИЧЕСКИЕ, СИНТАКСИЧЕСКИЕ И ФУНКЦИОНАЛЬНЫЕ ОСОБЕННОСТИ ДЕМОНСТРАТИВОВ МУАН. ПОКАЗАНО, ЧТО В СИСТЕМЕ ДЕМОНСТРАТИВОВ МУАН, НАСЧИТЫВАЮЩЕЙ ПЯТЬ ЭЛЕМЕНТОВ, ОКАЗЫВАЮТСЯ ЗАДЕЙСТВОВАНЫ СЛЕДУЮЩИЕ ПРИЗНАКИ: 1) СООТНЕСЁННОСТЬ С ГОВОРЯЩИМ, СОБЕСЕДНИКОМ ИЛИ ТРЕТЬИМ ЛИЦОМ; 2) НАХОЖДЕНИЕ В ЗОНЕ ВИДИМОСТИ ГОВОРЯЩЕГО ИЛИ СОБЕСЕДНИКА ИЛИ ВНЕ ЕЁ; 3) НАХОЖДЕНИЕ В КОНТАКТЕ ИЛИ ВНЕ КОНТАКТА С ТЕЛОМ ГОВОРЯщЕГО.

\section{INDEX}

Keywords : System of Demonstratives, Deixis, Mwan, Word Classes motsclesru ДЕМОНСТРАТИВЫ, ДЕЙКСИС, МУАН, КЛАССЫ ЛЕКСЕМ Mots-clés : demonstratifs, déixis, classes des mots Thèmes : mwan

\section{AUTEUR}

\section{ELENA PEREKHVALSKAYA}

St. Petersbourg, Institut de recherches linguistiques elenap96@gmail.com 